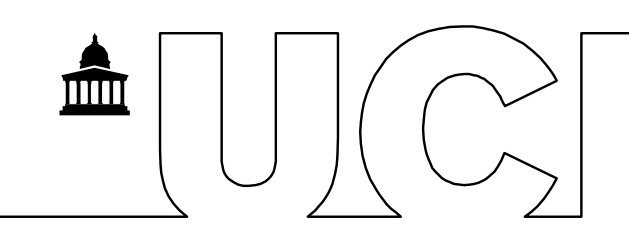

Alic, N; Partridge, L; (2015) Myc mouse and anti-ageing therapy. Trends in Endocrinology \& Metabolism, 26 (4) pp. 163-164. 10.1016/i.tem.2015.02.005. Downloaded from UCL Discovery:

http://discovery.ucl.ac.uk/1463170

\title{
ARTICLE
}

\section{Myc mouse and anti-ageing therapy}

Nazif Alic ${ }^{1}$ and Linda Partridge ${ }^{1,2}$ *

${ }^{1}$ Institute of Healthy Ageing, Department of Genetics, Evolution and Environment, University

College London, Darwin Building, Gower Street, London, WC1E 6BT, UK.

${ }^{2}$ Max Planck Institute for Biology of Ageing, Joseph-Stelzmann-Strasse 9b, 50931 Cologne, Germany.

*Corresponding author: Linda Partridge (I.partridge@ucl.ac.uk)

\begin{abstract}
Reduction in the expression and activity of a well-known proto-oncogene, Myc, has a beneficial effect on mouse health and survival to old age, in part independently of cancer impact, a recent study reveals. Is this new anti-ageing intervention pointing a way towards new treatments for age-related diseases?
\end{abstract}

\section{Text}

The time an organism stays alive (lifespan) and healthy (healthspan) is, for most animals, limited by an intrinsic process of deterioration that is ageing. In humans, age is the major risk factor for the predominant killer and chronic diseases, including cancer and cardiovascular disease, and for debilitating pathologies, such as neurodegeneration. The proportion of older people in many populations is rising fast, with individuals over 85 years old the most rapidly expanding age-group in developed countries ${ }^{1}$. Because the major burden of ill health is falling on older people, ameliorating the effects of ageing has become an urgent priority for research.

The hallmarks of ageing are becoming well delineated and are complex, but the process has proved to be malleable, because both healthspan and lifespan can be extended by genetic, environmental and pharmacological interventions ${ }^{2}$. Recent work by Hofmann, Zhao and coworkers in the journal Cell adds a new, and intriguing, intervention to the current anti-ageing arsenal, by showing that mice heterozygous for Myc loss-of-function have increased lifespan and healthspan ${ }^{3}$.

Investigation of Myc started in the late 1970s with the discovery that retrovirally induced chicken tumours contained a new viral oncogene, $v$-Myc. The host genome contained a version of this gene, the Myc proto-oncogene, which contributes to the aetiology of many human cancers. Myc encodes a transcriptional regulator controlling the expression of a large portion of the genome to direct cell growth, proliferation, tumorogenesis and stem cell function ${ }^{4}$. Hofmann, Sedivy and coworkers noticed that many of the cellular and organismal processes promoted by Myc are also present in ageing and age-related diseases, prompting them to examine ageing in a mouse where constitutively reduced activity of $M y c$ was achieved by deletion of one of its copies from the genome ${ }^{3}$. 
Median lifespan of $\mathrm{Myc}^{+-}$mice was extended relative to controls by $10.7 \%$ in males and $20.9 \%$ in females. The mutant mice also showed functional improvements, with lower levels of cardiac fibrosis, osteoporosis and more youthful levels of lipid metabolism and motor performance ${ }^{3}$. Such broad-spectrum protection against age-related changes argues that the extended healthy lifespan was not simply due to preservation of function of a specific tissue or prevention of a specific disease. Indeed, the incidence of the main cause of death, lymphoma, appeared to be unaffected in $\mathrm{Myc}^{+-}$mice, although their lymphomas had progressed and spread less by the time that they died ${ }^{3}$. Furthermore, reduced Myc activity was previously shown to extend lifespan in Drosophila ${ }^{5}$, an invertebrate model where death is unlikely due to cancer. The broadspectrum effects and evolutionary conservation of Myc function in longevity support its potential as a basis for anti-ageing therapy in humans.

Interestingly the Myc mutant mouse lacked some features of other long-lived mouse models, including altered content or distribution of fat, altered rates of cellular senescence, genotoxic stress or apoptosis, and not reduced fecundity. These phenotypes may therefore not contribute to increased lifespan or, alternatively, lowered Myc activity may act through different mechanisms to promote healthy ageing ${ }^{3}$. The transcriptome of $\mathrm{Myc}^{+/-}$mice showed preservation at later ages of youthful levels and patterns of expression of genes involved in lipid metabolism and immune function, both processes dysregulated during normal ageing. Myc is a major transcriptional regulator of ribosome biogenesis, and $\mathrm{Myc}^{+-}$mice showed reduced content of ribosomal proteins, rRNA and reduced rates of translation, features often associated with antiageing interventions ${ }^{3}$. The $\mathrm{Myc}^{+/}$mice also had a higher metabolic rate, were more active, and consumed more food and water than controls.

A relationship between reduced activity of $M y c$ and other interventions that increase healthspan is implied by its relationship with the Forkhead Box $\mathrm{O}$ (FoxO) transcription factor family members. FoxO activity can extend healthy lifespan in invertebrate model organisms, and variants of FoxO genes are robustly linked to human longevity, 6 . Interestingly, FoxO and Myc antagonise each other at numerous levels ${ }^{6}$, and it will be revealing to explore this relationship in the $\mathrm{Myc}^{+-}$mouse. The activation status of kinases that regulate FoxO members, such as AKT and $\mathrm{AMPK}$, were altered in the $\mathrm{Myc}^{+-}$mice, and they had lower circulating levels of IGF-1, either as a consequence of direct Myc action or of feedback in the signalling network. It will be informative to investigate the genetic interactions between partial loss-of-function in Myc and other longevity interventions.

Myc is notable for being the most highly amplified oncogene in human cancers ${ }^{4}$. The relationships between the mechanisms of cancer prevention and longevity assurance are complex $^{2}$ (Figure 1). For example, cellular senescence forms a potent tumour suppressive mechanism but it also contributes to ageing and ageing-related pathologies ${ }^{7}$, and a similar antagonism can be observed at the level of telomere maintenance ${ }^{2}$. On the other hand, several manipulations that moderately increase the activity of tumour supressors and those that block oncogenes often extend lifespan. Examples include components of pathways well known in the context of ageing, such as Pten in the insulin/IGF signalling cascade ${ }^{8}$, and genes mostly appreciated for their function in tumour suppression, such as the Ink4/Arf locus ${ }^{9}$, and now Myc ${ }^{3}$. In all cases, extension of lifespan is mostly independent of reduced cancer incidence. Similarly, rapamycin, an inhibitor of the TOR kinase, and a pharmacological treatment effective against ageing in a number of model organisms including mice, also has anti-cancer activity ${ }^{10}$.

This link importantly suggests that some drugs developed to treat cancer could also be used for the treatment of other ageing-related diseases (Figure 1). 


\section{Acknowledgements}

The work in LP's lab is supported by the Wellcome Trust and Max Planck.

\section{Figure caption}

Figure 1 Relationship between cancer, ageing and potential therapies.

The complex relationship between mechanisms that suppress or promote cancer and ageing raises the question whether therapies designed for cancer treatment can be modified to treat other diseases of ageing.

\section{References}

1. Christensen, K., et al. (2009) Ageing populations: the challenges ahead. Lancet 374, 1196-1208

2. Lopez-Otin, C., et al. (2013) The hallmarks of aging. Cell 153, 1194-1217

3. Hofmann, J.W., et al. (2015) Reduced Expression of MYC Increases Longevity and Enhances Healthspan. Cell 160, 477-488

4. Dang, C.V. (2012) MYC on the path to cancer. Cell 149, 22-35

5. Greer, C., et al. (2013) Myc-dependent genome instability and lifespan in Drosophila. PLoS One 8, e74641

6. Eijkelenboom, A., and Burgering, B.M. (2013) FOXOs: signalling integrators for homeostasis maintenance. Nat Rev Mol Cell Biol 14, 83-97

7. Campisi, J. (2013) Aging, cellular senescence, and cancer. Annu Rev Physiol 75, 685705

8. Ortega-Molina, A., et al. (2012) Pten positively regulates brown adipose function, energy expenditure, and longevity. Cell Metab 15, 382-394

9. Matheu, A., et al. (2009) Anti-aging activity of the Ink4/Arf locus. Aging Cell 8, 152-161

10. Lamming, D.W., et al. (2013) Rapalogs and mTOR inhibitors as anti-aging therapeutics. J Clin Invest 123, 980-989 